\title{
ÉTAT D'AVANCEMENT DES RECHERCHES SUR LA MODÉLISATION DE L'HABITAT DES POISSONS DES COURS D'EAU EN FRANCE.
}

\section{Y. SOUCHON}

CEMAGREF, Division Biologie des Ecosystèmes Aquatiques, Laboratoire d'Hydroécologie Quantitative, C.P. 220, 3 bis Quai Chauveau, 69336 LYON Cedex 09 (France).

\section{RÉSUMÉ}

Les recherches sur l'habitat physique des poissons n'ont été vraiment développées en France que dans les quinze dernières années. L'article en présente un bilan surtout orienté vers les méthodes de quantification qui incluent hydraulique, géomorphologie et biologie suivant la philosophie de I'IFIM (Instream Flow Incremental Methodology) proposée par l'US Fish and Wildlife Service, Fort Collins, Colorado, U.S.A. Plusieurs travaux ont été menés à bien. Ils concernent :

- le développement et le test des méthodes de description et de modélisation morphohydraulique à différentes échelles depuis le microhabitat jusqu'au tronçon de cours d'eau ;

- l'établissement de courbes de préférence d'habitat, essentiellement pour la truite fario à partir d'échantillonnages ponctuels à l'électricité, mais ce qui est plus original à partir de campagnes d'experts placés en situation dans un cours d'eau test ;

- des vérifications biologiques des hypothèses sous-jacentes de cette famille de méthodes, à savoir le lien entre l'habitat et la taille de la population de poissons. Ces tests ont nécessité des conditions expérimentales très strictes dans des cours d'eau exempts d'altérations (zones de référence).

Les méthodes développées rendent possible la mesure de la variabilité physique des cours d'eau. Elles répondent au souci d'évaluer et de prédire les impacts liés aux modifications hydrologiques avec la préoccupation de fournir des aides à la définition des débits environnementaux.

\section{DEVELOPMENT OF FISH HABITAT MODELIZATION IN FRENCH STREAMS IN RECENT YEARS.}

\section{SUMMARY}

An assessment of quantitative methodologies for fish habitat evaluation is presented.

By crossing hydraulics, geomorphology and biology, and following the IFIM philosophy (Fort Collins. US Fish and Wildlife Service, Colorado, USA), several results have been obtained :

- development and standardization of habitat descriptions at different spatial scales (microhabitat, mesohabitat, reach) ;

— evaluation of the habitat temporal variability using hydraulic simulations ; 
- development of habitat suitability curves or models from expert advice or from fish density and habitat data collected in streams

- biological tests of the methodology by comparing predicted versus observed brown trout biomass in reference streams.

This recent advance offers different tools for measuring the spatio-temporal variability of streams and rivers. This is of high interest for physical impact evaluation, in particular when the hydrological pattern is altered, i.e. definition of ecological flows.

\section{INTRODUCTION}

En France, les recherches sur l'habitat physique des poissons ont connu un certain essor depuis une quinzaine d'années parallèlement à l'utilisation accrue des cours d'eau à des fins de production hydroélectrique, d'irrigation, de prélèvement pour l'eau potable ou de lutte contre les crues.

La perception d'une dégradation généralisée des milieux a fait naître une sensibilité de protection, clairement illustrée par le texte de la loi relative à la pêche en eau douce de juin 1984 qui introduit diverses garanties de protection des écosystèmes aquatiques assorties d'obligations de résultats. Sont instaurés des débits minimums légaux dans les cours d'eau (article L.232-5 du Code Rural), un principe de libre circulation des poissons migrateurs (art. L.232-6) et des garanties par rapport aux travaux d'aménagement des cours d'eau (art. L.232-3). La nouvelle loi sur l'eau de janvier 1992 réaffirme à son tour la nécessité de préserver les milieux naturels aquatiques. Elle prévoit notamment la mise en place de schémas d'aménagement et de gestion des eaux (S.A.G.E.) par bassin ou ensemble de bassins pour harmoniser les différentes interventions humaines en fonction de la sensibilité écologique des milieux. D'où un besoin accru de connaissances sur le fonctionnement hydrodynamique des cours d'eau accompagné de méthodes et d'outils pour appréhender la sensibilité de l'habitat.

La première connaissance mobilisable dans ce domaine est déjà assez ancienne. Elle concerne l'organisation qualitative longitudinale des peuplements de poissons. Depuis HUET (1954) et VERNEAUX (1977a, 1977b) nous disposons de critères simples pour déterminer le peuplement de poissons qualitatif de stations de cours d'eau d'une région biogéographique donnée : pente et largeur de HUET sont complétées par température, section mouillée, distance aux sources et dureté totale par VERNEAUX. Le peuplement étudié est comparé au peuplement de référence de la même région pour déterminer l'existence d'éventuelles altérations. Plusieurs référentiels régionaux ont depuis été produits selon des principes voisins : Lorraine, MOUILLE, 1983 ; Drôme, WASSON et al., 1984 ; bassin de la Seine, Yonne, CEMAGREF, 1988 ; BELLIARD, 1994.

Tout comme les travaux nord-américains réalisés à l'échelle plus vaste des écorégions (HAWKES et al., 1986 ; ROHM et al., 1987), ces approches sont nécessaires comme cadrage préalable de toute étude diagnostique. N'étant pas bâties sur des relations de causes à effets clairement établies, elles s'avèrent insuffisantes pour permettre l'identification précise des facteurs responsables de dégradations. Ce ne sont en aucun cas des outils prédictifs. D'où l'ambition des recherches plus récentes sur l'habitat de travailler à des niveaux d'organisation modifiables par l'action humaine (morphologie et hydrologie) et sur des facteurs directement perceptibles par les organismes (substrat, hauteur d'eau, vitesse du courant, végétation).

Notre propos est de faire le point sur l'expérience acquise en France au cours des dix dernières années dans ce domaine, en abordant successivement :

- la notion de profil écologique utile pour transcrire les exigences spatiales des espèces. Différents types de modèles d'habitat sont possibles et plusieurs voies ont été explorées. Leur développement doit tenir compte des possibilités de couplage avec des approches physiques de description du comportement hydrodynamique des cours d'eau ; 
- la notion de capacité d'accueil physique potentielle d'un cours d'eau pour une ou plusieurs espèces de poissons. Le cheminement adopté consiste à simuler le fonctionnement physique de tronçons homogènes de cours d'eau en choisissant des stations représentatives dont l'hydrodynamique est étudiée finement. A l'aide des profils ou modèles écologiques, chaque portion discrète d'espace aquatique fait alors l'objet d'une analyse de confort potentiel par espèce et stade de développement. Nous discutons de cette pratique plus connue sous l'appellation quelque peu réductrice de méthode des microhabitats.

\section{CONSTRUCTION DES PROFILS ÉCOLOGIQUES D'HABITAT}

L'habitat aquatique est ici défini par la combinaison de paramètres essentiellement physiques qui déterminent en chaque point d'un cours d'eau l'espace de vie des poissons. Les exigences spatiales de ces organismes varient en fonction des espèces, de leur stade de développement et des phases de leur cycle vital (reproduction, nutrition, abri, repos). L'originalité de l'habitat aquatique est d'être composé d'une partie fixe, le substrat, structuré par la dynamique fluviale et d'une partie mobile, l'écoulement, décrit par la vitesse du courant et la hauteur d'eau. S'y ajoutent des éléments de complexification comme la végétation aquatique ou les débris ligneux.

Plusieurs approches ont été mises en oeuvre pour construire des profils écologiques d'habitat des poissons.

\section{Information bibliographique}

Les compilations bibliographiques sont toujours délicates car les informations sont relatives à des écosystèmes et des assemblages faunistiques très variés pour des données recueillies selon des protocoles peu standardisés. Toutefois, elles conduisent à dresser les grands traits des exigences spatiales des espèces. Ainsi, d'après la littérature, GRANDMOTTET (1983) a pu esquisser les principales exigences des poissons d'eau douce d'Europe de l'Ouest, en retenant une description assez générale par espèce. A titre d'exemple, la vandoise (Leuciscus leuciscus, L. 1758) est décrite comme se reproduisant préférentiellement sur des alluvions de diamètre $3-10 \mathrm{~cm}$ ou $3-15 \mathrm{~cm}$, où la vitesse est comprise entre 20 et $50 \mathrm{~cm} / \mathrm{s}$, dans le lit principal des cours d'eau. Elle s'alimente dans la masse d'eau aux mêmes vitesses et se repose ou s'abrite plutôt dans les herbiers du chenal dans des vitesses nulles à faibles $(0$ à $20 \mathrm{~cm} / \mathrm{s}$ ).

Cette connaissance très générale permet déjà d'identifier les zones des cours d'eau les plus propices pour certaines espèces, de les repérer cartographiquement (procédure des schémas départementaux de vocation piscicole, SOUCHON \& TROCHERIE, 1990) et parfois de justifier leur protection (par exemple, arrêté de biotope pour la protection intégrale d'une frayère).

Dans cette même catégorie, peuvent être classées les courbes dites de "préférence d'habitat" transcrites sous forme de fonctions normées entre 0 et 1 selon l'affinité de l'espèce ou du stade pour un paramètre donné (exemple, la hauteur d'eau) après interprétation bibliographique. Les premières courbes proposées par WATERS (1976) et BOVEE et COCHNAUER (1977) étaient de ce type.

De même, les courbes du saumon atlantique (Salmo salar, L. 1758) utilisées dans l'étude Orne (COUROT, 1988) et dans l'étude Allier (SOUCHON \& VALENTIN, 1991) étaient essentiellement livresques.

\section{Le recours direct à des experts}

Il est possible de dresser des profils d'habitat en faisant appel à un panel d'experts réunis autour d'une table ou consultés épistolairement (Delphi technique : CRANCE, 1988). 

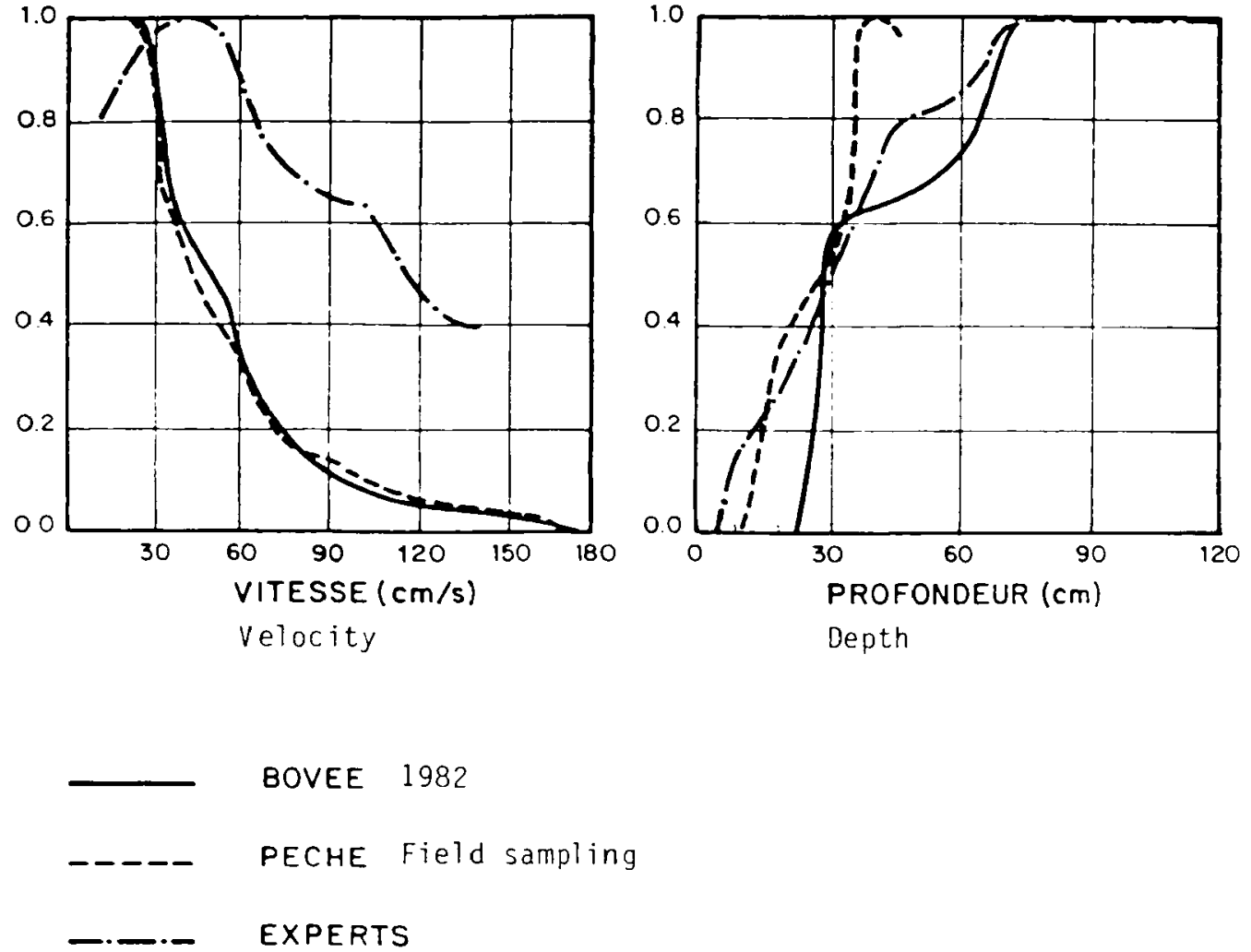

Figure 1 : Comparaison des courbes de préférence d'habitat bâties selon trois méthodes (BOVEE, 1982 ; pêches sur le site ; experts) (extrait de CHAVEROCHE et al., 1987).

Figure 1 : Different habitat preference curves from three origins : BOVEE, 1982 ; local sampling ; expert advices (CHAVEROCHE et al., 1987).

Plus originale a été l'expérience menée en France, à l'initiative d'EDF, Etudes et Recherches, qui a réuni 15 experts d'horizons géographiques différents au bord d'un cours d'eau à truites. Le but était de bâtir des courbes d'habitat de la truite fario (Salmo trutta fario, L. 1758) à partir des réponses des experts qui devaient se prononcer sur l'habitabilité de microzones matérialisées précisément et dont les caractéristiques physiques avaient été décrites séparément. Les résultats (fig. 1) ont montré que la technique était performante et conduisait le plus souvent à des courbes proches de celles déjà publiées (CHAVEROCHE et al., 1987). Un fort décalage entre courbe expérimentale et courbe expert pour la vitesse (fig.1) montre toutefois que certains paramètres sont mal appréhendés et mal quantifiés directement par les experts. II semble donc prudent de réserver ce type d'approche aux espèces pour lesquelles peu de données expérimentales ont encore pu être rassemblées.

\section{Les courbes expérimentales à partir de données réelles}

A condition de réunir suffisamment d'informations sur le positionnement précis des poissons dans leur habitat, il est possible d'exprimer les relations observées de différentes manières.

Tout d'abord, plusieurs variantes existent selon :

- la technique d'investigation : pêche électrique, observation de surface, observation subaquatique, radiotélémétrie, 
- l'espace pris en compte : point focal, point de pêche, ambiance, microzone,

- les paramètres dont la valeur peut être estimée (technique des Echantillons Ponctuels d'Abondance, NELVA et al., 1979) ou mesurée. Cette deuxième option est préférable lorsque l'on veut coupler ces modèles avec une description morphohydraulique ;

- le traitement de données :

- monovarié par paramètre en calculant des histogrammes de densités normés par la valeur maximum (ORTH \& MAUGHAN, 1982) : il s'agit de courbes d'utilisation. On parle de courbes de préférence lorsque ces courbes sont corrigées par la disponibilité de l'habitat au moment de l'échantillonnage.

- bivarié : VOOS et al., 1981 cités par BOVEE (1985) ou LAMBERT \& HANSON (1989) pour la vitesse et la hauteur d'eau (salmonidés de la côte ouest des USA). Ce type de traitement pourrait apporter une première réponse aux critiques des modèles existants au sujet de la dépendance des variables (MATHUR et al., 1985).

- multivarié qui autorise la prise en compte des interactions entre variables, l'intégration de nouveaux paramètres physiques ou biotiques (présence d'autres espèces ou stades) (BAIN et al., 1988 ; SOUCHON et al., 1989 ; VALENTIN et al., 1991 ; POUILLY et SOUCHON, 1993).

Les données expérimentales recueillies et traitées en France à ce jour concernent principalement la truite fario avec des courbes expérimentales pour les rivières du Massif Central et du sud-est (FRAGNOUD, 1987) et celles du sud-ouest (BELAUD et al., 1988). Un premier jeu de courbes a également été produit pour le barbeau fluviatile (Barbus barbus, $L$. 1758), le chevaine (Leuciscus cephalus, L. 1758), le gardon (Rutilus rutilus, L. 1758) et le goujon (Gobio gobio, L. 1758) à partir d'une série d'échantillonnages dans le Rhône, l'Ardèche et l'Ain (POUILLY et SOUCHON, 1990). Il vient d'être complété par les travaux de POUILLY et SOUCHON (1994).

Tableau I : Espèces de poissons pour lesquelles des profils d'habitat monovariés et multivariés ont été établis (POUILLY et SOUCHON, 1994).

Table I : Fish species with multivariate habitat profiles (POUILLY et SOUCHON, 1994).

NOM SCIENTIFIQUE

Salmo trutta fario
Barbus barbus
Rutilus rutilus
Leuciscus cephalus
Leuciscus (telestes) soufia
Alburnus alburnu:
Alburnoïdes bip nctatus
Phoxinus phoxinus
Gobio gobio
Noemacheilus barbatulus
Perca fluviatilis

Salmo trutta fario

Barbus barbus

ilus rutilus

Leuciscus cephalus

Leuciscus (telestes) soufia

Alburnus alburnu:

Alburnoïdes bip nctatus

Phoxinus phoxinus

Noemacheilus, barbatulus

Perca fluviatilis

L'information disponible actuellement est synthétisée dans le Tableau I.

\section{Vers une nouvelle génération de profils écologiques d'habitat}

Différentes possibilités de prendre en compte l'habitat des poissons sont donc offertes sous forme de modèles plus ou moins frustes. Devant cette multiplicité, il devient nécessaire de rechercher des critères de validation : 
[a] Peut-on essayer de comparer la robustesse des différents profils et leur validité statistique par des tests de prédiction?

[b] Comment répondre aux interrogations sur la dépendance des variables, sur l'intégration d'un espace plus complexe réellement perçu par le poisson?

Par exemple pour BACHMAN (1984) étudiant la truite, le seul point focal n'est pas suffisant pour expliquer son positionnement : la sélection des postes de chasse se fait en fonction de gradients de vitesse par juxtaposition d'un refuge hydraulique (bloc) et d'une veine à forte vitesse pourvoyeuse de nourriture (dérive).

[a] La robustesse de la prédiction de plusieurs modèles a été testée sur un jeu de données constitué par un échantillon de 100 microzones ou ambiances d'une surface variant de 1 à $10 \mathrm{~m}^{2}$ (MALAVOI, 1990) dans un cours d'eau du Massif Central, la Boralde de St Chely (détails dans CAPRA, 1990).

Sur chaque ambiance, les truites ont été pêchées et six points de mesures ont été relevés (hauteur d'eau, vitesse, substrat).

Les données ont été analysées de plusieurs manières :

- courbes d'utilisations (dénommées Aveyron);

- modèle multivarié : Analyse Factorielle des Correspondances (AFC) intergroupes (CHESSEL \& DOLEDEC, 1991) suivie d'une analyse discriminante sur les coordonnées des deux premiers axes factoriels (classes de densité prédites 0 adulte $/ \mathrm{m}^{2}, 1$ à 2 adultes $/ \mathrm{m}^{2}, 3$ adultes et plus).

Pour chaque microzone, il est possible de calculer une classe de densité estimée à partir de chacune des méthodes. Cette estimation est comparée aux valeurs de densités réelles pour :

— les courbes monovariées généralistes de BOVEE (1982);

- les courbes monovariées d'utilisation du site (Aveyron);

- les courbes monovariées d'utilisation d'un autre site (Jura);

- le modèle multivarié (AFC).

Les résultats non publiés ont montré que la prédiction de la densité des adultes est à peu près équivalente avec les trois types de courbes monovariées: faible capacité de prédiction de la classe 1 à 2 adultes (21 à 26\%), capacité moyenne à bonne pour les classes 0 et plus de 3 adultes $(55 \%$ à $83 \%$ ). II y a peu de différences entre les courbes des trois origines, ce qui ne corrobore pas l'idée généralement admise selon laquelle les courbes établies sur le site sont plus performantes. La prédiction obtenue grâce au traitement multivarié est systématiquement meilleure (classe $0: 65 \%$, classe 1 ou $2: 58 \%$, classe $3: 100 \%$ ) et permet d'obtenir un gain de précision moyen de $10 \%$ par comparaison avec les courbes monovariées (test de $\chi^{2}$ ).

Le recours à ce type de traitement multivarié est donc certainement une voie prometteuse.

[b] La prise en compte d'une plus grande complexité physique est rendue possible grâce au traitement multivarié des données. Il permet à la fois de tenir compte des interactions possibles entre variables "de base" déjà choisies et ajout de nouvelles variables (position dans le chenal, abri, type de végétation) (SOUCHON et al., 1989). Le choix de l'échelle spatiale n'est pas neutre. L'ambiance, échelle intermédiaire entre le microhabitat strict et le faciès, offre une nouvelle dimension à explorer pour se rapprocher des repères spatiaux élémentaires réellement perçus par les individus.

Il faut par ailleurs veiller à bien représenter tous les habitats nécessaires à l'accomplissement du cycle vital des espèces, l'échantillonnage classique par pêche électrique pouvant omettre des phases importantes. Cette difficulté peut être levée par des observations directes du comportement du poisson (plongée ou surface) ou par recours au radiopistage (thèse de BARAS, 1992 sur le barbeau fluviatile). 


\section{COUPLAGE DES MODĖLES PHYSIQUES AVEC LES MODĖLES BIOLOGIQUES : VERS L'ESTIMATION D'UNE CAPACITÉ D'ACCUEIL PHYSIQUE POTENTIELLE}

La collaboration entre biologistes et hydrauliciens a permis d'échafauder une méthode dite des microhabitats, qui définit une capacité d'accueil physique potentielle des cours d'eau en couplant une description et une modélisation physiques à des modèles biologiques d'habitat (IFIM*). Fort Collins, Etats-Unis (BOVEE, 1982). C'est certainement la méthode la plus utilisée à l'heure actuelle (REISER et al., 1989), mais elle est aussi très détractée (MATHUR et al., 1985 ; SCOTT \& SHIRVELL, 1987). Les critiques portent sur l'interdépendance des variables choisies non prises en considération lors de l'emploi de courbes de préférence monovariées et sur le manque de validation biologique de l'hypothèse fondatrice selon laquelle I'habitat constitue l'ultime facteur limitant structurant les populations de poissons.

En partie pour répondre à ces critiques, des équipes d'Etudes et Recherches d'EDF et du CEMAGREF Lyon ont testé l'applicabilité de la méthode des microhabitats en France. Nous détaillons ci-après l'expérience acquise au sein de ces équipes. Certaines adaptations méthodologiques ont été proposées (SOUCHON et al., 1989):

- révision du profil d'habitat de la truite proposé par BOVEE et COCHNAUER (1977) pour la hauteur d'eau ;

- mise en place d'un protocole de description topographique et hydraulique des cours d'eau (MALAVOI \& SOUCHON, 1989) ou d'un protocole faisant appel à plusieurs campagnes de mesure (SABATON et al., 1994). Les deux procédures ont été récemment rassemblées et décrites dans le protocole SABATON et al. (1994);

- révision des formules utilisées dans la modélisation hydraulique (SOUCHON et al., 1989).

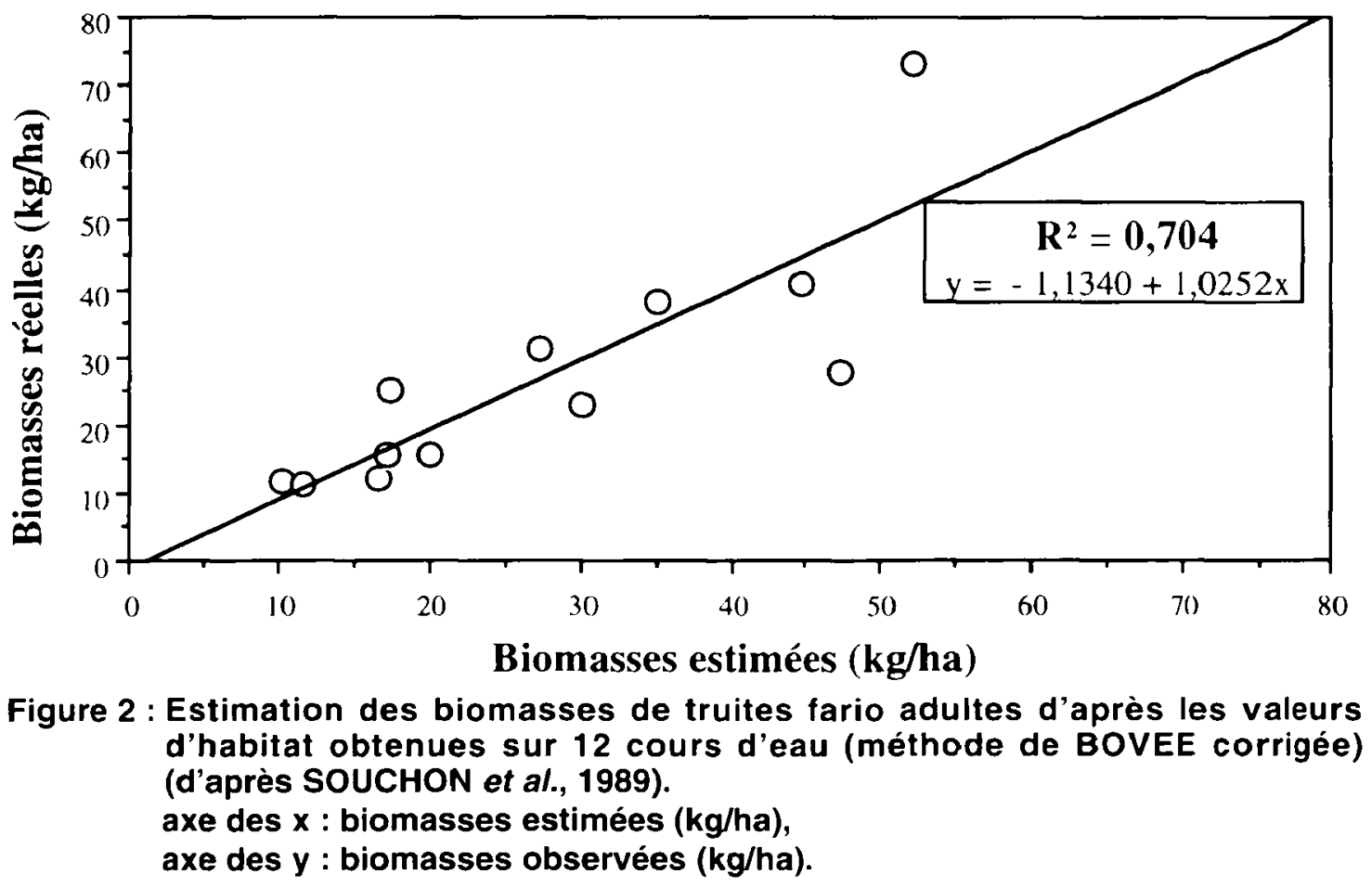

Figure 2 : Theoretical (modelized) versus observed biomass in 12 streams (modified BOVEE, habitat preference curve) (from SOUCHON et al., 1989).

$x$ axis : modelized biomass $(\mathrm{kg} / \mathrm{ha})$,

$y$ axis : observed biomass ( $k g / h a)$.

(*) IFIM : Instream Flow Incremental Methodology. 
Dans un souci de validation générale de la démarche, le CEMAGREF a appliqué la méthode à une quinzaine de cours d'eau de référence exempts de pollution chimique (Classe 1A), non alevinés et peu fréquentés par les pêcheurs (accessibilité très réduite, loin des axes de circulation routière). La population de truite de ces secteurs de cours d'eau a été échantillonnée deux années de suite. Les résultats ont été encourageants puisqu'une bonne concordance a été constatée entre biomasse réelle et biomasse estimée par la méthode des microhabitats (fig. 2) (stade adulte limitant, habitat limitant équivalent au minimum de Surface Pondérée Utile calculé sur la base des débits moyens mensuels - SOUCHON et al., 1989). II est fondamental de raisonner de façon dynamique en reconstituant les chroniques réelles d'habitat pour les débits connus de façon à identifier les évènements susceptibles d'influencer la population (BOVEE, 1988). Nombre de critiques sont nées à la suite d'études conduites sur un pas de temps trop court (liaison stock-habitat instantanée), alors que la population adulte qui contribue fortement à la biomasse résulte d'évènements biologiques, physiques ou chimiques antérieurs à l'observation. Les sites incriminés ne sont d'ailleurs pas toujours exempts d'anthropisation. D'autre part, l'habitat apparaît comme une condition nécessaire mais pas toujours suffisante pour expliquer la situation atteinte par une population. La nourriture, la prédation ou même la compétition interspécifique pour des rivières au peuplement plus complexe, peuvent être des facteurs déterminants du stock en place (WATERS, 1982 ; BAGLINIERE \& CHAMPIGNEULLE, 1982).

Suite à cette première validation pour les cours d'eau à forte énergie et à peuplement de truite, la méthode a été employée comme aide au choix des débits réservés à l'aval des prises d'eau. II apparaît généralement que le $1 / 40^{\mathrm{e}}$ du module (valeur plancher de la loi de 1984 pour les ouvrages anciens) est une valeur qui pénalise très fortement $(>50 \%$ ) la capacité d'accueil potentielle d'une population de truite et que le $1 / 10^{\mathrm{e}}$ du module (valeur plancher pour les nouveaux ouvrages) reste significativement pénalisante (entre 20 et $50 \%$ du niveau optimal). Ce jugement doit être relativisé par rapport au type de cours d'eau, et à sa morphologie, les secteurs à galets-graviers et large lit moyen étant plus sensibles que les secteurs en gorge avec des mouilles bien établies.

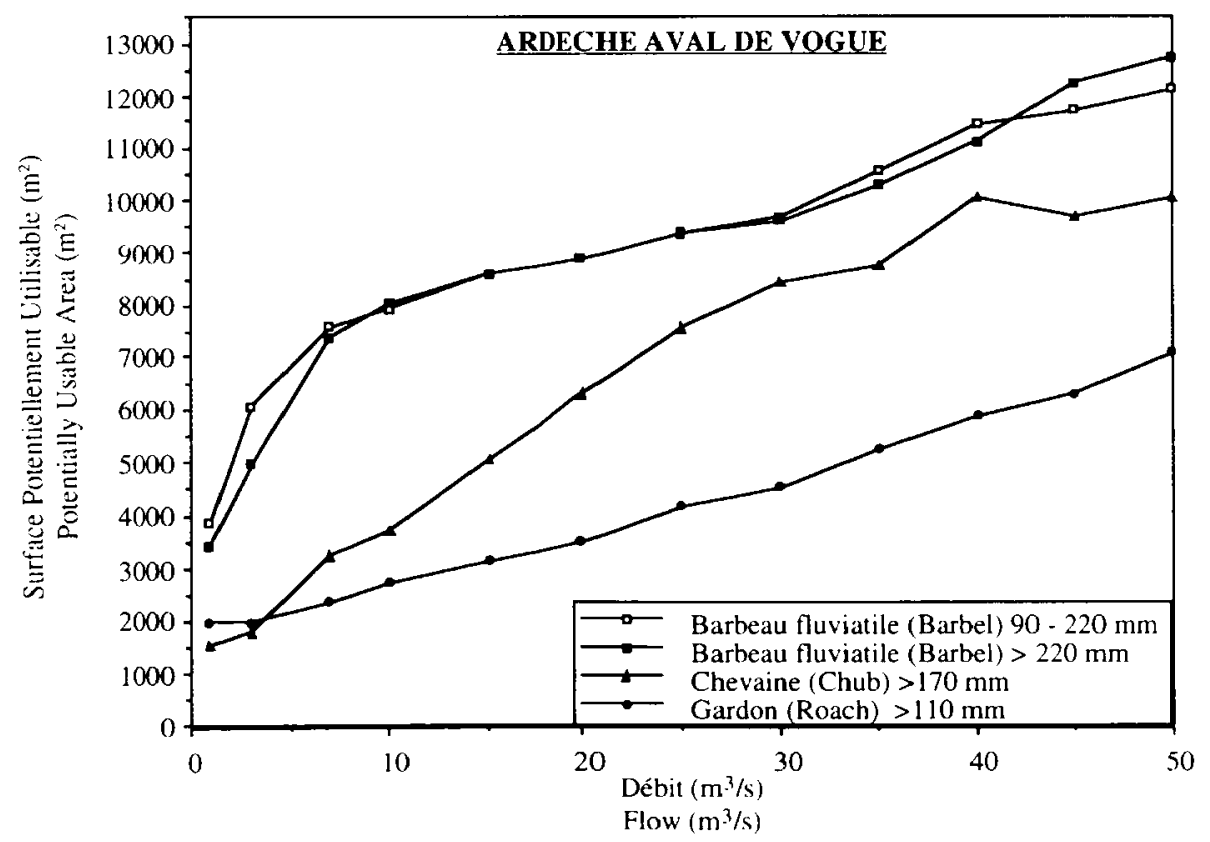

Figure 3 : Evolution des Surfaces Potentiellement Utilisables en fonction du débit calculées sur une station de la rivière Ardèche (modèles multivariés) d'après POUILLY \& SOUCHON (1994).

Figure 3 : Potentially Usable Area versus flow on one study site of the Ardèche river (multivariate models) from POUILLY \& SOUCHON (1994). 
La communauté scientifique bénéficie désormais d'un retour d'expérience d'une cinquantaine de cas traités pour lesquels peuvent être produites des analyses stationnelles bien utiles pour apprécier le comportement physique et l'évolution de l'habitat (fig. 3).

L'interprétation des valeurs de capacité d'accueil en fonction du débit n'est pas toujours aisée et doit s'assortir de quelques précautions. En raison du nombre d'étapes et d'options possibles, il est nécessaire de bien mentionner quel cheminement a été choisi.

Il est important de :

- préciser les choix des espèces cibles ;

- choisir judicieusement la représentativité des stations d'étude et de raisonner par faciès avec au moins 3 transects de mesure dans chaque faciès;

- comparer le régime des débits après aménagement au régime naturel en gardant à l'esprit cette référence ;

- tenir compte de la dynamique des autres compartiments que le poisson : durée, fréquence, calendrier des épisodes hydrologiques pour la ripisylve, l'équilibre morphologique, le nettoyage des substrats, supports des frayères des salmonidés en particulier. Ce type de méthodologie n'est pas exclusivement réservé à la détermination de débits minimums. D'autres applications sont possibles pour mieux cerner, par exemple, l'impact des modifications morphologiques ou mieux comprendre l'effet des variations de débit à court terme imposées par l'exploitation en éclusées hydroélectriques (programme en cours, EDF Etudes et Recherches, SABATON \& LAUTERS, 1992 ; VALENTIN et al., 1994).

La méthode des microhabitats appliquée judicieusement constitue par conséquent un bon outil d'analyse dynamique de la sensibilité physique d'un cours d'eau. Lorsque les tests biologiques sont suffisamment avancés et probants, une capacité physique d'accueil potentielle peut alors être estimée avec une certaine sécurité.

Après avoir analysé finement le comportement physique d'une station d'un cours d'eau à l'échelle de vie des organismes et de réalisation des processus, il est légitime de s'interroger sur la façon de généraliser les résultats à un linéaire plus grand.

Le faciès morphodynamique ou mésohabitat (FRISSEL et al., 1986 ; MALAVOI, 1989 ; KERSHNER \& SNIDER, 1992) apparaît comme une échelle de transition logique et commode pour au moins trois raisons:

— sa réalité géomorphologique qui permet une identification sur le terrain standardisable ;

- sa reconnaissance comme structure-clé du fonctionnement écologique par nombre de biologistes (BAGLINIERE \& CHAMPIGNEULLE, 1982 ; OSWOOD \& BARBER, 1982) qui y ont associé des valeurs de capacité d'accueil et de production pour les salmonidés à l'étiage ;

- un premier niveau de globalisation des simulations numériques de capacité d'accueil obtenues par transect.

Parce qu'il est possible de reconnaître par tronçon de cours d'eau les successions de structures morphologiques élémentaires, l'étude d'une séquence de faciès représentatifs peut rendre compte de tout un linéaire de cours d'eau. La sectorisation morphologique apparaît alors comme une base de travail préalable à toute étude biologique (fig. 4). La généralisation doit toutefois être assortie de garde-fous en vérifiant si possible le niveau de variabilité inter faciès en termes d'habitabilité.

L'analyse des caractéristiques et de la sensibilité de la séquence de faciès échantillons peut ensuite être réalisée avec différents niveaux de précision, selon les moyens et les objectifs des gestionnaires :

— classification des cours d'eau ; 


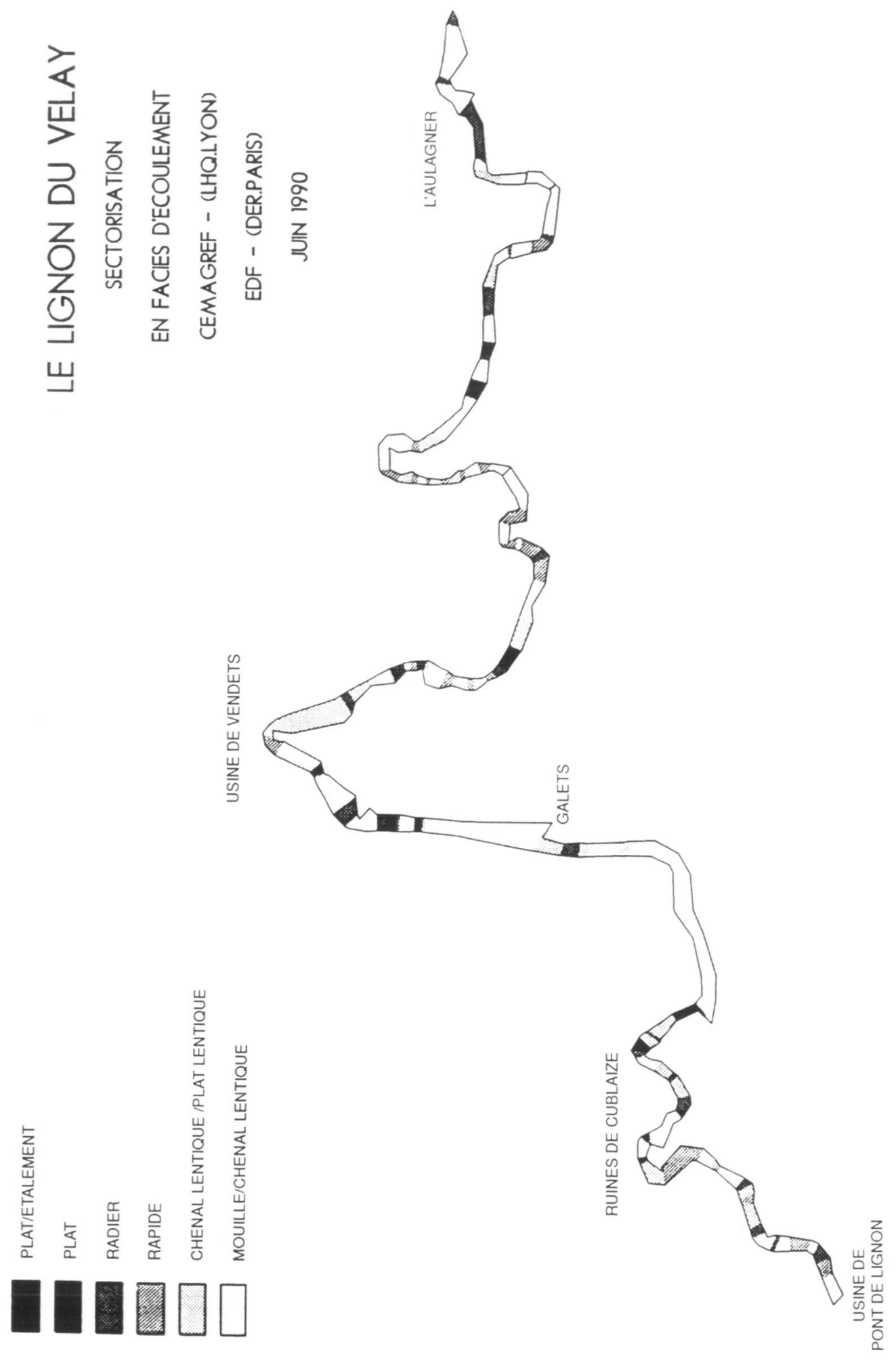

Figure 4 : Exemple de sectorisation d'un cours d'eau en faciès préalable à un calcul de simulation de capacité d'accueil physique (SOUCHON et al., 1990).

Figure 4 : Stream sectorization by mesohabitat before Physical Habitat Simulation (from SOUCHON et al., 1990). 
- estimation de leur capacité de production en certaines espèces (ex. saumon atlantique, INRA Rennes, BAGLINIERE \& CHAMPIGNEULLE, 1982);

- identification des faciès de frayères potentielles ou de grossissement des jeunes (études CSP $^{*}$, Rhin, Dordogne, Garonne) ;

- sensibilité des faciès aux modifications hydrauliques ou morphologiques en recourant aux méthodes de simulation numérique, avec extrapolation possible à l'ensemble d'un tronçon de cours d'eau.

\section{CONCLUSION}

L'approche pluridisciplinaire qui a caractérisé les recherches sur l'habitat au cours de la dernière décennie explique vraisemblablement les progrès accomplis. Elle a abouti à la mise à disposition d'outils de compréhension et de diagnostic plus performants.

Des compléments sont à attendre des axes de recherche qui restent encore à explorer dans cet esprit. II s'agit, par exemple, des travaux coordonnés dans le cadre des programmes PIRE** :

- étude affinée des facteurs de macrorépartition des espèces de poissons à l'échelle des bassins fluviaux (PONT \& DE MERONA, 1991);

- modélisation de l'utilisation spatio-temporelle de l'habitat par les poissons : exemples de la truite, de l'ombre commun et du barbeau fluviatile (SOUCHON \& GAUDIN, 1991). L'accent est mis sur la variabilité naturelle de l'habitat.

De façon complémentaire, c'est à EDF (Etudes et Recherches) que l'on doit l'animation de travaux plus spécifiques sur les effets des modifications hydrauliques à court terme (programme éclusées hydroélectriques, SABATON \& LAUTERS, 1992).

Dans ce dernier cas, la réponse écologique est étudiée en fonction d'une variabilité hydraulique imposée par différents modes d'exploitation énergétique des cours d'eau.

A une échéance relativement proche, il est clair que nous pourrons disposer d'outils d'analyse de la dynamique d'habitat beaucoup plus performants.

Est-il besoin de rappeler qu'ils ne constitueront malgré tout qu'une des facettes de la connaissance des écosystèmes lotiques.

\section{REMERCIEMENTS}

Ce texte relate les travaux relevant de plusieurs programmes de recherche français sur l'habitat: "Débits réservés et éclusées" (Etudes et Recherches EDF) ; «PIR habitatpoisson» (Ministère de l'Environnement, Direction de l'eau, SRETIE).

Concernant les recherches conduites au CEMAGREF, l'auteur tient à remercier chaleureusement tous les membres du Laboratoire d'Hydroécologie Quantitative (Division BEA, Lyon) qui ont contribué activement et dans une ambiance exceptionnelle à ces travaux.

L'auteur remercie également le Comité de lecture de la revue, qui a permis d'améliorer le texte initial.

(*) CSP : Conseil Supérieur de la Pêche.

$\left.{ }^{\star *}\right)$ PIRE : Programme Interdisciplinaire de Recherche sur l'Environnement (CNRS). 


\section{BIBLIOGRAPHIE}

BACHMAN R.A., 1984. Foraging behavior of free ranging wild and hatchery brown trout in a stream. Trans. Am. Fish. Soc., 113, 1-32.

BAGLINIERE J.L., CHAMPIGNEULLE A., 1982. Densité des populations de truite commune (Salmo trutta, L.) et de juvéniles de saumon atlantique (Salmo salar, L.) sur le cours principal du Scorff (Bretagne) : preferendums physiques et variations annuelles (1976-1980). Acta Oecologica Oecol. Applic., 3 (3), 241-256.

BAIN M.B., FINN J.T., BOOKE H.E., 1988. Stream flow regulation and fish community structure. Ecology, 69 (2), 382-392.

BARAS E., 1992. Contribution à l'étude des stratégies d'occupation du temps et de l'espace chez un poisson téléostéen dulcicole, le barbeau fluviatile Barbus barbus (L.). Etude par radiopistage, pêche à l'électricité et observation directe. Thèse de Doctorat en Sciences. Université de Liège : 214 p. + annexes.

BELAUD A., CHAVEROCHE P., LIM P., SABATON C., 1988. Courbes de preferenda d'habitat de la truite fario (Salmo trutta fario, L.) dans des rivières du sud-ouest de la France. Rapport EDF E31/88-40 : 20 p.

BELLIARD J., 1994. Structuration des peuplements ichtyologiques du bassin de la Seine. Actes du colloque franco-québécois sur l'intégration des technologies modernes à la gestion des poissons dulcicoles et amphihalins. 31 mai, 1, 2, 3 juin 1992.

BOVEE K.D., COCHNAUER T., 1977. Development of weighted criteria, probability-of-use curves for instream flow assessments : fisheries. Instream Flow Information Paper, 3. U.S. Fish and Wildlife Service Volley. FWS/OBS-77/63. 38 p.

BOVEE K.D., 1982. A guide to stream habitat analysis using the Instream Flow Incremental Methodology. Instream Flow Information Paper $n^{\circ} 12$. FWS/OBS 82/26. Western Energy and Land Use Team, U.S. Fish and Wildlife Service, Ft. Collins, Colorado : 248 p.

BOVEE K.D., 1985. Evaluation of the effects of hydropeaking on aquatic macroinvertebrates using PHABSIM. Small hydro/Fisheries symposium, Denver, Colorado (May 1-3, 1985) : $6 \mathrm{p}$.

BOVEE K.D., 1988. Use of the instream flow incremental methodology to evaluate influences of microhabitat variability on trout populations in four Colorado streams. Proceedings of the 68th annual conference, Western association of Fish and Wildlife Agencies, Albuquerque : $31 \mathrm{p}$.

CAPRA H., 1990. Modélisation des préférences d'habitat de la truite fario (Salmo trutta fario L., 1758). Intégration de la variabilité locale des paramètres physiques. DEA Analyse et modélisation des systèmes biologiques, rapport technique : $31 p+$ annexes.

CEMAGREF, 1988. Etude de I'Yonne. Rapport d'étude AFB Seine Normandie/CEMAGREF Div. Qual. Eaux, Pêche \& Piscic. : 42 p. + annexes.

CHAVEROCHE P., GRAS R., SABATON C., 1987. Quinze experts analysent l'habitat de la truite fario. Note technique EDF, Direction des Etudes et Recherches : $28 p+$ annexes.

CHESSEL D., DOLEDEC S.,1991. ADE version 3.1. Hypercard stacks and Quickbasic microsoft programme library for the analyse of environmental data. URA CNRS 1451 , Univ. LYON I, 43 Bd du 11 novembre 1918, 69622 VILLEURBANNE Cedex, France.

COUROT A., 1988. Débit réservé de l'Orne à Rabodanges. Evaluation de la qualité d'habitat piscicole en fonction du débit. Rapport Direction des Etudes et Recherches, EDF, HE 31, 88/07, 23 p. + annexes.

CRANCE J.H., 1988. Results of the use of the Delphi technique for developing category I habitat suitability criteria for Redbreast sunfish. Proceedings of a workshop on the development and evaluation of habitat suitability criteria. U.S. Dept of the Interior Fish and Wildlife Service. Biological report $88(11): 102-126$. 
FRAGNOUD E., 1987. Préférences d'habitat de la truite fario (Salmo trutta fario L., 1758) en rivière. (Quelques cours d'eau du sud-est de la France). Thèse 3ème cycle, CEMAGREF DQEPP-LHQ, Université C. Bernard Lyon I: 398 p.

FRISSEL C.A., LISS W.J., WARREN C.E., HURLEY M.D., 1986. A hierarchical framework for stream classification : viewing streams in a watershed context. Environmental Management, 10, 199-214.

GRANDMOTTET J.P., 1983. Principales exigences des téléostéens dulcicoles vis-à-vis de l'habitat aquatique. Annales Sci. Univ. Besançon. Biol. Anim., 4ème série, $4:$ 3-32.

HAWKES C.L., MILLER D.L., LAYHER W.G., 1986. Fish ecoregions of Kansas : stream fish assemblage patterns and associated environmental correlates. Environmental Biology of Fishes, 17 (4), 267-279.

HUET M., 1954. Biologie, profils en long et en travers des eaux courantes. Bull. Fr. Piscic., $175,41-53$.

KERSHNER J.L., SNIDER W.M., 1992. Importance of a habitat level classification system to design Instream Flow studies. In BOON P.J., CALOW P., PETTS G.E. (Eds), River Conservation and Management, John Wiley \& Sons Ltd, 179-193.

LAMBERT T.R., HANSON D.F., 1989. Development of habitat suitability criteria for trout in small streams. Regulated rivers : research and management, $3: 291-303$.

MALAVOI J.R., 1989. Typologie des faciès d'écoulement ou unités morphodynamiques des cours d'eau à haute énergie. Bull. Fr. Pêche \& Piscic., 315, 189-210.

MALAVOI J.R., SOUCHON Y., 1989. Méthodologie de description et quantifications des variables morphodynamiques d'un cours d'eau à fond caillouteux. Exemple d'une station de la Filière (Haute-Savoie). Revue Géographique de Lyon, 64 (4), 252-259.

MALAVOI J.R., 1990. Méthodologie de sectorisation et de description des cours d'eau à pente forte et moyenne. Application à une gestion intégrée des écosystèmes d'eau courante. Thèse Université Jean Moulin, Lyon III, CEMAGREF DQEPP-LHQ, $555 \mathrm{p}$ t annexes.

MATHUR D., BASON W.H., PURDY E.J.Jr., SILVER C.A., 1985. A critique of the Instream Flow incremental Methodology. Can. J. Fish. Aquat. Sci., 42, 825-831.

MOUILLE J., 1983. Esquisse typologique des peuplements ichtyologiques des rivières lorraines 1973-1982. DEA en Sciences Agronomiques. Génie de l'Env. et Aménagement ENAIA Nancy: 86 p.+ carte.

NELVA A., PERSAT H., CHESSEL D., 1979. Une nouvelle méthode d'étude des peuplements ichtyologiques dans les grands cours d'eau par échantillonnage ponctuel d'abondance. C.R. Acad. Sc., 289 D, 1295-1298.

NESTLER J.M., MILHOUS R.T., LAYZER J.B., 1989. Instream habitat modelling techniques, in Gore \& Petts (Eds). Alternatives in regulated River Management, 344 p. : 295-315.

ORTH D.J., MAUGHAN O.E., 1982. Evaluation of the incremental methodology for recommending Instream Flows for fishes. Trans. Am. Fish. Soc., 111, 413-445.

OSWOOD M.E., BARBER W.E., 1982. Assessment of fish habitat in streams : goals, constraints, and a new technique. Fisheries, 7 (3), 8-11.

PONT D., DE MERONA B., 1991. Signification des échelles spatio-temporelles dans la variabilité des peuplements piscicoles des grands cours d'eau. PIR Habitat-poisson, Rapport de programme, $20 \mathrm{p}$.

POUILLY M., SOUCHON Y., 1990. Schéma de vocation piscicole du fleuve Rhône. Tronçon court-circuité de Montélimar. Simulation des capacités d'habitat potentiel des poissons. Rapport Lab. Hydroéc. Quant. CEMAGREF DQEPP Lyon, $20 p+$ annexes. 
POUILLY M., SOUCHON Y., 1993. Simulation de l'habitat physique du barbeau fluviatile (Barbus barbus L.). Cahiers d'éthologie, 13, 135-138.

POUILLY M., SOUCHON Y., 1994. Méthode de simulation de la capacité d'accueil physique des cours d'eau pour les poissons. Application aux cours d'eau de taille moyenne (module supérieur à $30 \mathrm{~m}^{3} / \mathrm{s}$ ). Aide à la détermination d'un débit réservé dans le cadre de l'application de l'article L 232-5 du Code Rural. CEMAGREF BEA Lyon/LHQ et Ministère de l'Environnement, Direction de l'eau, Rapport final convention $91289,61 \mathrm{p}$.

REISER D.W., WESCHE T.A., ESTES C., 1989. Status of Instream Flow legislation and practices in North America. Fisheries, 14 (2), 22-29.

ROHM C.M., GIESE J.W., BENNETT C.C., 1987. Evaluation of an aquatic Ecoregion classification of streams in Arkansas. Journal of Freshwater Ecology, 4 (1), 127-140.

SABATON C., LAUTERS F., 1992. Impacts éventuels sur l'écosystème aquatique de la gestion "par éclusées" de certains ouvrages hydroélectriques. Programme de recherche. Note technique EDF $n^{\circ} \mathrm{HE} 31 / 92-04,12 \mathrm{p}$.

SABATON C., VALENTIN S., SOUCHON Y., 1994. La méthode des microhabitats. Protocoles d'application. EDF/DER, CEMAGREF BEA LYON/LHQ. 26 p.

SCOTT D., SHIRVELL C.S., 1987. A critique of the Instream Flow Incremental Methodology and observations on flow determination in New Zealand. In Kemper J.B. and Craig J. (Ed.), Regulated streams advances in Ecology. Plenum Press, New-york : 27-43.

SOUCHON Y., TROCHERIE F., FRAGNOUD E., LACOMBE C., 1989. Les modèles numériques des microhabitats des poissons, application et nouveaux développements. Revue des sciences de l'eau, 2, 807-830.

SOUCHON Y., MALAVOI J.R., COUROT A., MIQUEL J., 1990. Le Lignon du Velay, du barrage de Lavalette jusqu'au confluent avec la Loire : simulation de l'habitat des poissons. Aide à la détermination d'un débit réservé. Rapport CEMAGREF, DQEPP LHQ-EDF, DER Chatou-SRAE Auvergne, $88 \mathrm{p}$.

SOUCHON Y., TROCHERIE F., 1990. Technical aspects of French legislation dealing with freshwater fisheries (June 1984) : "Fisheries orientation schemes" and «Fishery resources management plans". In W.L.T. Van DENSEN, B. STEIMETZ \& R.H. HUGUES (Eds). Management of Freshwater Fisheries. Proceedings of a symposium organized by the EIFAC Göteborg, Sweden, 31 may-3 june 1988 : 190-214.

SOUCHON Y., GAUDIN P., 1991. Modélisation de l'utilisation spatio-temporelle de l'habitat par les poissons : exemples de la truite, de l'ombre commun et du barbeau fluviatile. PIR poisson. Programme «modèles d'habitat» 1992-1994. Rapport de présentation, $34 \mathrm{p}$.

SOUCHON Y., VALENTIN S., 1991. L'Allier du barrage de St-Etienne du Vigan jusqu'à Langeac. Simulation de l'habitat physique des salmonidés. Rapport CEMAGREF Lyon, BEA-LHQ : $75 \mathrm{p}$.

STALNAKER C.B., 1979. The use of habitat structure preferenda for establishing flow regimes necessary for maintenance of fish habitat. In The Ecology of Regulated Streams, Ward and Stanford eds., Plenum Press, New-York, p. 326-337.

VALENTIN S., CAPRA H., SOUCHON Y., WASSON J.G., 1991. Evaluation de la capacité d'accueil physique des cours d'eau vis-à-vis de la truite fario. Prise en compte de facteurs complémentaires : diversité de l'habitat et instabilité du débit. Rapport EDF, DER-CEMAGREF Lyon BEA-LHQ. Convention 247466 : 35 p.

VALENTIN S., SOUCHON Y., WASSON J.G., 1994. Evaluation of hydropeaking effects on fish community and habitat. Rehabilitation of Freshwater Fisheries. I. G. COWX (eds), Blackwell Scientific publication, London, 138-151. 
VERNEAUX J., 1977a. Biotypologie de l'écosystème "eau courante". Détermination approchée de l'appartenance typologique d'un peuplement ichtyologique. C.R. Acad. Sc. Paris, 284 D : 675-678.

VERNEAUX J., 1977b. Biotypologie de l'écosystème "eau courante". Déterminisme approché de la structure biotypologique. C.R. Acad. Sci. Paris, 284 D, 77-79.

WASSON J.G., PIALOT D., SOUCHON Y., ZYLBERBLATT M., DA COSTA G., 1984. Approche quantitative de l'impact écologique des aménagements de cours d'eau. Comm. Symp. CECPI-FAO, Aarhus, Dk. Doc. CEMAGREF, Div. Qual. Eaux, Pêche \& Pisc. - Lyon : $41 \mathrm{p}$.

WATERS B.F., 1976. A methodology for evaluating the effects of different streamflows on salmonid habitat. In ORSBORN \& ALLMAN. Instream Flow needs Symposium vol. II. Am. Fish. Soc., 254-266.

WATERS T.F., 1982. Annual production by a stream brook charr population and by its principal invertebrate food. Env. Biol. Fish., 7 (2), 165-170. 